\title{
On-orbit performance of the Spitzer Space Telescope
}

\author{
Thomas L. Roellig, Michael W. Werner, David B. \\ Gallagher, William R. Irace, Giovanni G. Fazio, et al.
}

Thomas L. Roellig, Michael W. Werner, David B. Gallagher, William R. Irace, Giovanni G. Fazio, James R. Houck, George H. Rieke, Robert K. Wilson, Thomas Soifer, "On-orbit performance of the Spitzer Space Telescope," Proc. SPIE 5487, Optical, Infrared, and Millimeter Space Telescopes, (12 October 2004); doi: 10.1117/12.551037

Event: SPIE Astronomical Telescopes + Instrumentation, 2004, Glasgow, United Kingdom 


\title{
On-orbit performance of the Spitzer Space Telescope
}

\author{
Thomas Roellig*a ${ }^{\mathrm{a}}$, Michael Werner ${ }^{\mathrm{b}}$, David Gallagher ${ }^{\mathrm{b}}$, William Irace ${ }^{\mathrm{b}}$, Giovanni Fazio ${ }^{\mathrm{c}}$, James \\ Houck $^{\mathrm{d}}$, George Rieke ${ }^{\mathrm{e}}$, Robert Wilson ${ }^{\mathrm{b}}$, Thomas Soifer ${ }^{\mathrm{f}}$ \\ ${ }^{a}$ NASA Ames Research Center, MS 245-6,Moffett Field, CA, USA 94035 \\ ${ }^{\mathrm{b}}$ Jet Propulsion Laboratory, MS 264-767, 1600 Oak Grove Ave., Pasadena, CA, USA 91109 \\ ${ }^{\mathrm{c}}$ Smithsonian Astrophysical Observatory, MS 65, 60 Garden St., Cambridge, MA, USA 02138 \\ ${ }^{\mathrm{d}}$ Department of Astronomy, 220 Space Sciences Bldg., Cornell University, Ithaca, NY, USA 14853 \\ 'University of Arizona, Steward Observatory, 933 N. Cherry Ave., Tucson, AZ, USA 85721 \\ ${ }^{\mathrm{f}}$ Spitzer Science Center - Caltech University, MS 314-6, Keith Spaulding Bldg., Pasadena, CA, \\ USA 91125
}

\begin{abstract}
The Spitzer Space Telescope (formally known as SIRTF) was successfully launched on August 25, 2003, and has completed its initial in-orbit checkout and science validation and calibration period. The measured performance of the observatory has met or exceeded all of its high-level requirements, it entered normal operations in January 2004, and is returning high-quality science data. A superfluid-helium cooled $85 \mathrm{~cm}$ diameter telescope provides extremely low infrared backgrounds and feeds three science instruments covering wavelengths ranging from 3.6 to 160 microns. The telescope optical quality is excellent, providing diffraction-limited performance down to wavelengths below 6.5 microns. Based on the first helium mass and boil-off rate measurements, a cryogenic lifetime in excess of 5 years is expected. This presentation will provide a summary of the overall performance of the observatory, with an emphasis on those performance parameters that have the greatest impact on its ultimate science return.
\end{abstract}

Keywords: Spitzer Mission, Infrared, Astronomy, Telescope

\section{INTRODUCTION}

The Spitzer Space Telescope (formerly known as the Space Infrared Telescope Facility, or SIRTF) is the fourth and last of NASA's Great Observatories. Spitzer provides mid and far-infrared observational capabilities complementing the wavelengths accessible by the other Great Observatories: the Compton Gamma-Ray Telescope, the Hubble Space Telescope, and the Chandra X-Ray Observatory. Spitzer was launched on August 25, 2003, has completed an initial period of on-orbit checkout and science performance validation and is now in normal operation and returning excellent science data. A compendium of the early science results from the Spitzer mission can be found in an issue of the Astrophysical Journal Supplement Series ${ }^{1}$ that has been devoted to the first Spitzer science results. Examples of some other early science results can also be found elsewhere in these proceedings. ${ }^{2}$

The Spitzer flight components are shown in Figure 1 and certain top-level characteristics are given in Table 1. In order to reach its required infrared sensitivity the Spitzer instruments and the telescope had to be capable of operation at temperatures below $5.5 \mathrm{~K}$ - a requirement that entered into all aspects of the facility and mission design, including the choice of orbit. The observatory is divided into a warm assembly comprising the spacecraft bus structure and its contents and the solar power generation panels (hereafter referred to as the spacecraft), and a cold assembly (hereafter referred to as the Cryogenic Telescope Assembly or CTA), comprising the telescope, cryostat, and the focal-plane instruments. These two assemblies are connected to each other by a truss structure of eight very low thermalconductivity struts, while thermal radiation from the warm to cold components is minimized through the use of radiation shields and aluminized-mylar thermal blankets located between the two. Further details of the observatory flight hardware design are given below and in other references ${ }^{3,4}$.

* Thomas.L.Roellig@nasa.gov: phone 1650 604-6426 


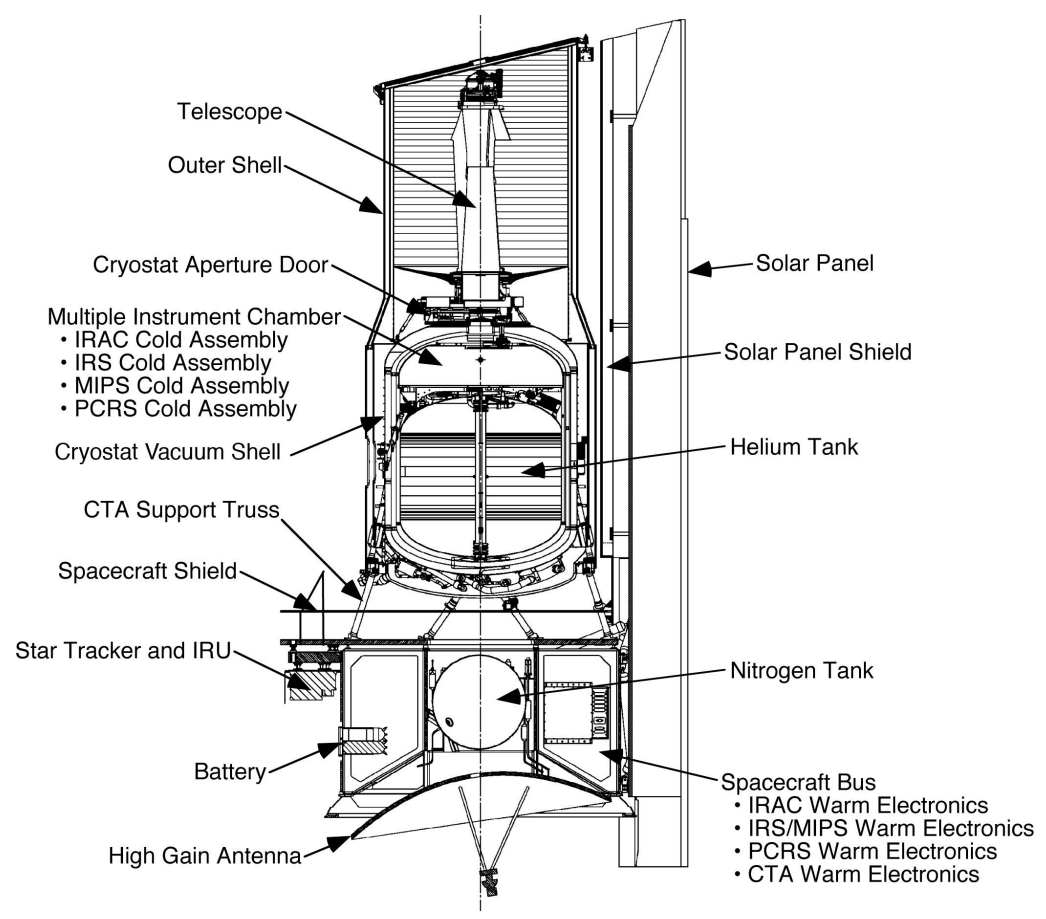

Figure 1 A cut-away view of the Spitzer observatory. In this figure the dust cover over the telescope aperture is shown in place before its ejection, which occurred four days after launch.

Table 1

Top-Level Observatory Parameters

\begin{tabular}{ll}
\hline Total Observatory Mass at Launch & $861 \mathrm{~kg}$ \\
Approximate Observatory Dimensions (height x diameter) & $4.5 \mathrm{~m} \mathrm{x} ø 2.1 \mathrm{~m}$ \\
Average Operating Power & $375 \mathrm{~W}$ \\
Solar Array Generating Capacity at Launch & $500 \mathrm{~W}$ \\
Nitrogen Reaction Control Gas at Launch & $15.59 \mathrm{~kg}$ \\
Estimated Reaction Control Gas Lifetime & 17 years \\
Mass Memory Capacity & 2 GBytes \\
Telescope Primary Diameter & $0.85 \mathrm{~m}$ \\
Telescope Central Areal Obscuration & $14.2 \%$ \\
Superfluid Helium at Launch & 337 liters \\
Estimated Nominal Cryogenic Lifetime & 5.3 years \\
Data Downlink Rate - High-Gain Antenna at up to $8.7 \times 10^{7} \mathrm{~km}$ distance & $2.2 \mathrm{Mbps}$ \\
Command Uplink Rate & $2 \mathrm{kbps}$ \\
Command and Telemetry Frequency & $\mathrm{X}$-band
\end{tabular}

\subsection{Spacecraft description}

The Spitzer spacecraft was developed by Lockheed-Martin and is responsible for the power generation, pointing and momentum control, data processing and storage, and telecommunications functions and hosts the warm electronics for the three scientific instruments. The spacecraft structure itself is constructed from graphite composite and aluminum 
honeycomb material and is octagonally-shaped ${ }^{5}$. In order to minimize the heat input from the solar array, which is Spitzer's warmest component, the array support structure is cantilevered from the spacecraft structure alone without any physical connection to the cold CTA. The approximately 1,500 wires running between the warm spacecraft and the cold CTA were constructed of very low thermal-conductivity materials and carefully heat-sunk so that they caused minimal parasitic heat input. The spacecraft avionics are all fully redundant, as are all of the components of the Pointing and Control System (PCS) and Reaction Control System (RCS) discussed below. The Spitzer command and datahandling $(\mathrm{C} \& \mathrm{DH})$ system is based around a redundant pair of RAD6000 computers operating at $20 \mathrm{MHz}$ and with 128 Mbytes of volatile RAM. The 16 Gbits of onboard mass memory storage is sized to the science instrument data rate to accommodate one missed downlink pass with the Deep Space Network (DSN) without causing any data overflow.

The Spitzer PCS components include reaction wheels, gyroscopes, and star trackers. The four reaction wheel assemblies are mounted in a pyramid arrangement, providing redundancy against a single wheel failure. Telescope slew control is provided through the gyro loop, while the star trackers are used to null out the gyro biases and provide stable pointing for longer science observations. The Spitzer thermal environment is particularly benign with the orbit described below. Even so, thermal variations can cause misalignment between the star trackers mounted on the warm spacecraft structure and cold telescope bore-sight. In order to track the variations in the star tracker-telescope alignment, a redundant pair of visible light sensors (Pointing Calibration Reference Sensors; PCRS) are located in the telescope focal plane as an integral part of the $\mathrm{PCS}^{6}$. With the Spitzer thermal design it is very important that one side of the CTA be facing the sun at all times. As a result the PCS and the C\&DH have been carefully designed so that Spitzer always operates within an Operational Pointing Zone (OPZ), with the telescope bore-sight staying within $80^{\circ}$ and $120^{\circ}$ of the spacecraft-sun vector and the observatory roll limited to $\pm 2^{\circ}$ around the telescope bore-sight. In addition to ground modeling checks and position checks within the spacecraft PCS software, a set of Wide-Angle Sun Sensors (WASS) is hard-wired into the spacecraft fault protection system to ensure that Spitzer is never positioned outside of the OPZ. Accumulated angular momentum is removed through a redundant set of 6 ambient-temperature, pressurized-nitrogen gas thrusters. This design allows accumulated momentum removal well outside the Earth's magnetic field while the nitrogen gas plume does not affect the infrared environment and the sensitive CTA thermal control surfaces.

\subsection{Cryogenic Telescope Assembly description}

The Spitzer CTA was developed by Ball Aerospace Corporation and consists of the telescope assembly, the cryogenic portions of the three science instruments, a superfluid helium dewar, and various thermal shields. The Spitzer thermal design is somewhat unusual in that most of the mass of the CTA, including the telescope and its baffles, is external to the cryostat vacuum shell and is launched at ambient temperature and only begins cooling when on-orbit. This allows for a much smaller vacuum pressure vessel and a smaller total observatory mass than would a design based on the more conventional architecture used in the earlier IRAS and ISO missions, where the telescope was launched cold inside a large vacuum shell. The components of the CTA external to the cryostat are cooled after launch by a combination of radiation and helium boil-off vapor. This degree of radiative cooling would not be possible in a low-earth orbit and was the major driver in the selection of the orbit described below. A very low-temperature CTA outer shell is critical to the achievement of a long mission lifetime with a small cryostat, but requires excellent thermal isolation between warm and cold components as well as tight restrictions on the orientation of the flight hardware with respect to the Sun. The CTA outer shell is painted with high-emissivity black paint on the side that faces away from the Sun and is polished aluminum on the sides that face the thermal shields, solar array, and warm spacecraft components. With the Spitzer design employing a smaller cryostat containing just the science instruments some sort of aperture needs to be opened after launch to allow light to enter the science focal plane. In Spitzer this functionality was realized with a removable vacuum window that was opened a few days after launch, once the telescope temperature had fallen sufficiently so that there would be minimal outgassing condensation on the cold instrument optics. This window was constructed of gold film-coated sapphire to allow the cold instruments to perform visible and near-infrared alignment of the CTA optical train on the ground without having to deploy the window prior to launch. A dust cover over the telescope aperture that was ejected 4.9 days after launch and 0.9 days prior to the opening of the cryostat window was the only other deployable mechanism on the Spitzer observatory.

The telescope optics and metering structure are constructed entirely of beryllium, minimizing changes in both the telescope prescription and its alignment with the focal plane as the telescope cools on-orbit. A moveable secondary constrained to move only parallel to the optical axis was installed to compensate for any changes in focus due to gravity 
release and uncertainties in the ground measurements of the telescope focus. The telescope optics are not cooled directly by the helium bath but are thermally coupled to the cryostat vacuum shell, which is cooled by the superfluid helium boil-off vapor. The higher the boil-off rate, the colder the telescope. The telescope temperature necessary to keep thermal emission from the optics negligible compared to the natural zodiacal background decreases with wavelength, reaching 5.6K for the $160 \mu \mathrm{m}$ channel in the Multiband Infrared Photometer for Spitzer (MIPS) instrument. MIPS itself does not dissipate enough power in its cold assemblies to provide the necessary boil-off rate for this temperature; a small adjustable heater in the cryogen provides a few extra milli-watts of power if needed. The helium liquid-gas interface is constrained within a porous plug phase separator, preventing rapid helium coolant loss.

\subsection{Science instrument descriptions}

The infrared light collected by the Spitzer telescope is fed to three science instruments via pick-off mirrors located in the telescope focal plane. Two of the instruments, the Infrared Array Camera (IRAC, Principal Investigator G. Fazio, SAO) and the Multiband Infrared Photometer for Spitzer (MIPS, Principal Investigator G. Rieke, University of Arizona), are designed as imaging instruments, although the MIPS also has a spectral energy distribution (SED) channel for very low-resolution spectroscopy. The third instrument, the Infrared Spectrograph (IRS, Principal Investigator J. Houck, Cornell University) provides low and moderate-resolution spectroscopic capabilities, although it has two small imaging peak-up apertures that can also be used for imaging. A short summary of the instrument capabilities is given in Table 2 and more details can be found in other papers in this volume and other references ${ }^{7,8,9}$.

Table 2

Summary of the Spitzer Science Instruments

\begin{tabular}{|c|c|c|}
\hline rument & Field of View & $\begin{array}{c}\text { Wavelength Range } \\
(\mu \mathrm{m})\end{array}$ \\
\hline
\end{tabular}

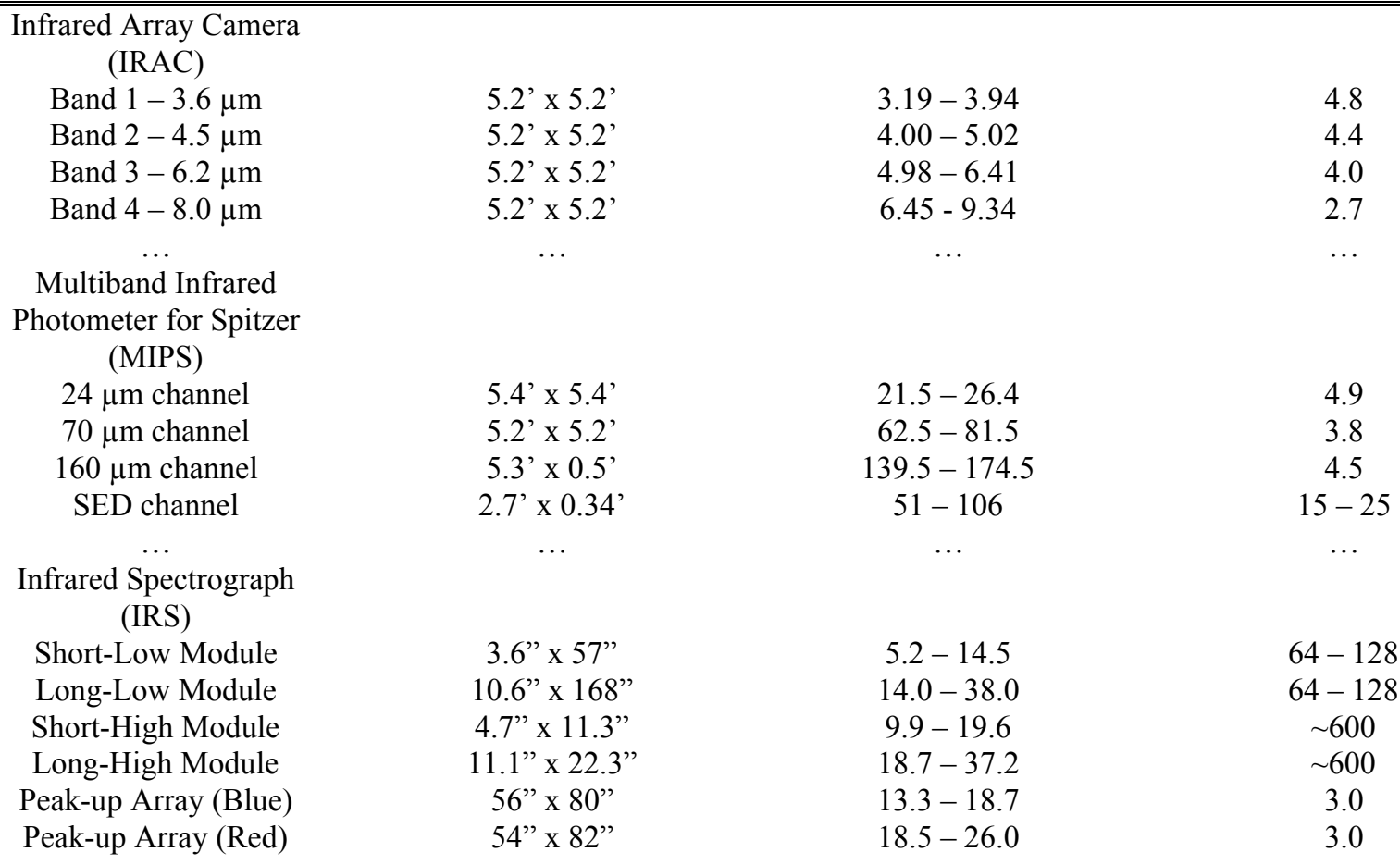

\subsection{Spitzer orbit and operations description}

In order to provide the benign thermal environment necessary for proper radiative cooling the Spitzer Space Telescope was launched into an Earth-trailing solar orbit ${ }^{10}$. As of May 19, 2004 Spitzer was trailing $12.4 \times 10^{6} \mathrm{~km}$ behind the Earth. As seen from Earth, Spitzer recedes at about $1.8 \times 10^{7} \mathrm{~km}$ per year and will reach a distance of $9.3 \times 10^{7} \mathrm{~km}$ in five 
years. Operationally, the orbit permits excellent sky viewing and observing efficiency. Figure 2 shows a diagram of the allowed observational areas of the sky while staying within the OPZ constraints. Finally, while in a solar orbit the observatory is not affected by the charged particles in the Earth's Van Allen radiation belts.
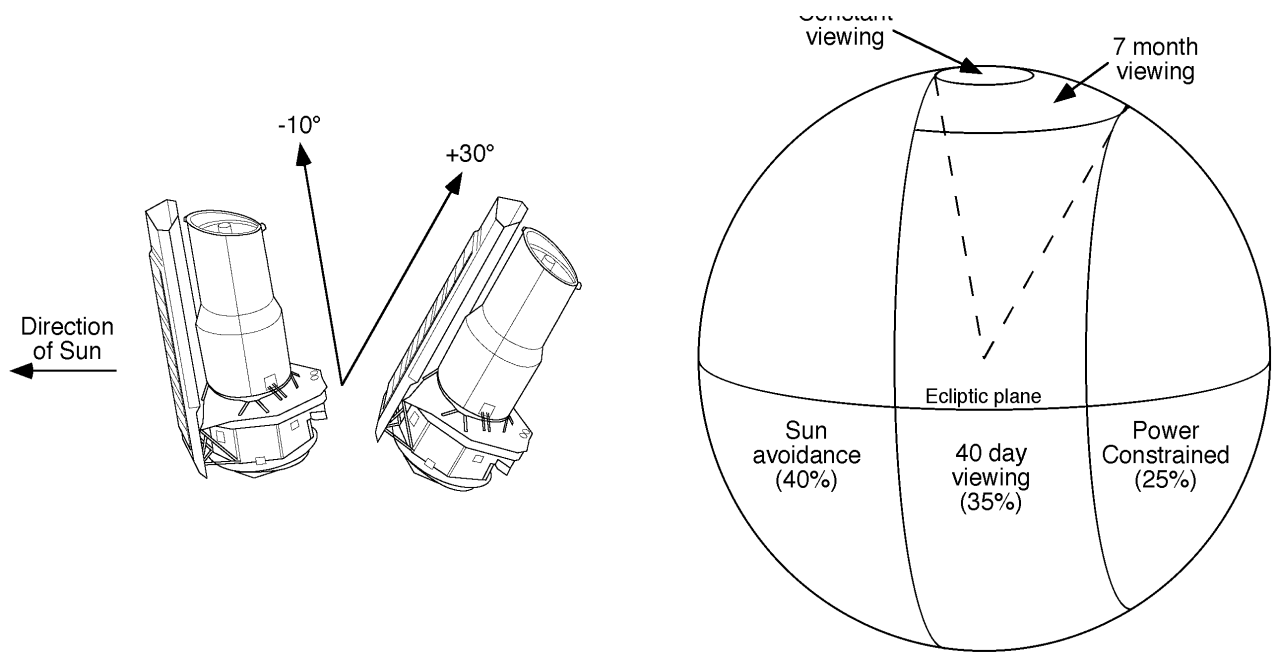

Figure 2. A diagram of the instantaneous sky visibility while staying within the Spitzer OPZ. Two regions near the ecliptic poles (the Constant Viewing Zones, or CVZ) are always visible from Spitzer. Sky locations near the ecliptic equator are visible for 40 days out of each 6-month period.

The orbit has two disadvantages. Firstly, as Spitzer moves away from the Earth, the power margins for data transmission and command communication decrease, forcing changes in communication strategy and, eventually, somewhat reduced efficiency as more time is spent communicating with the ground stations. The data are transmitted to the antennae of the Deep Space Network by orienting the spacecraft so that the fixed X-band antenna located on the bottom of the spacecraft shown in Figure 1 is pointed at the Earth. Our current data transmission strategy of two $~ 45$ minute passes per day at a data rate of $2.2 \mathrm{Mbps}$ is robust for at least the first 2.5 years of the mission Secondly, outside of the protection of the Earth's magnetosphere Spitzer is more susceptible to solar proton storms and is subject to a higher quiescent galactic cosmic rate flux. However, as mentioned above Spitzer is also free from the daily passages through the South Atlantic Anomaly which provide the main radiation dose in Low Earth Orbit.

Although the collected data are downlinked to the DSN twice a day, the onboard sequence of science observation commands is only refreshed once per week. Science operations and the science observing timeline are planned by the staff at the Spitzer Science Center and then sent to the Spitzer Mission Operations Center at JPL for transmission to the spacecraft. In order to minimize the thermal loads on the cryogen as well as the loads on the spacecraft C\&DH and power systems, only one of the three Spitzer science instruments is powered on at a time. Because the cryogen usage is dominated by the instrument power dissipation, there would be little benefit to parallel operations.

\section{ON-ORBIT PERFORMANCE}

The Spitzer observatory was launched from Kennedy Space Center on August 25, 2003 on a Delta 7920H launch vehicle and began a 90-day period of in-orbit checkout and scientific performance validation. We have been pleased to find that in almost every respect the on-orbit performance of the observatory meets or exceeds predictions. The most important on-orbit performance characteristics are listed below.

\subsection{Optical performance}

Prior to launch the Spitzer telescope secondary was moved to the position for predicted best-focus under the conditions of zero gravity and its planned cryogenic operating temperature. As the telescope cooled on-orbit the focus changes with temperature were tracked and the decision was made to initiate a campaign of three secondary-mirror focus moves 
to remove the remaining focus errors. As it turned out, after the second focus move the telescope was determined to have meet its focus requirements and the third secondary-mirror movement was dropped ${ }^{11}$. After the telescope was properly focused measurements using the IRAC instrument showed that it provides diffraction-limited performance at wavelengths greater than $5.5 \mu \mathrm{m}$, considerably better performance than the requirement of $6.5 \mu \mathrm{m}$. Figure 3 shows the measured Spitzer on-orbit point spread function. A more detailed discussion of the Spitzer optical image quality including more figures of the point-spread function and images of stellar objects can be found in Gehrz et al ${ }^{12}$. Measurements using all three science instruments also determined that the instruments are confocal to within each instruments' depth of focus.
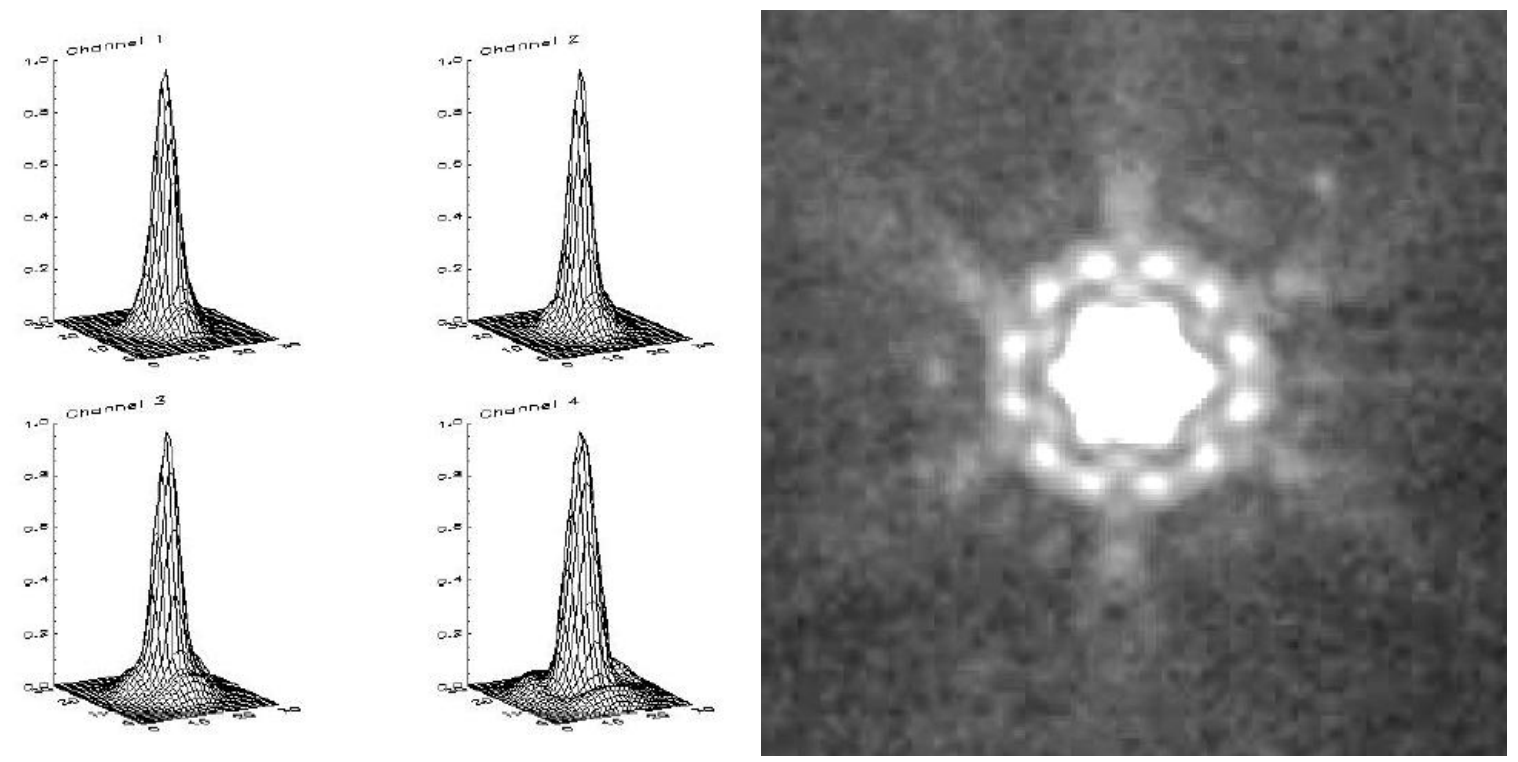

Figure 3. The measured on-orbit point spread functions for the four IRAC channels (left) and a MIPS $24 \mu \mathrm{m}$ image of a point source (right). The six-fold diffraction symmetry in the MIPS image is due to the triangular symmetry in the Spitzer telescope secondarymirror metering tower.

\subsection{Cryogenic performance}

Spitzer requires the unique low-thermal environment of space in order to function properly; the environment both permits the necessary radiative cooling and provides the low backgrounds to allow the science instruments to achieve their high sensitivities. This environment was difficult or impossible to simulate on the ground so that - notwithstanding a major pre-launch test, analysis, and modeling effort - there was some uncertainty in how well the observatory would perform in orbit. As it turned out the outermost CTA shield - the outer shell - attained its final temperature of 34-34.5 K solely by radiative cooling, and the telescope cooled to its operating temperature of $5.6 \mathrm{~K}$ in 41 days. The mass of the helium remaining after the initial cool down was measured to be $43.4 \pm 1.8 \mathrm{~kg}$ by applying a heat pulse to the helium bath 57 days after launch and measuring the accompanying temperature rise. The quantity of helium that was measured was significantly greater than the predicted mass of $37 \mathrm{~kg}$ for this phase of the mission, which should directly translate into increased mission lifetime. Two factors entered into this greater-than-expected helium mass, a smaller maximum bath temperature during the launch phase and faster-than-expected CTA shield cooling. To maintain the telescope at 5.5 $\mathrm{K}$ requires a total heat of $5.6 \mathrm{~mW}$ to the helium bath, leading to a boil-off of approximately $22 \mathrm{~g}$ of helium per day. For a fixed telescope temperature, these nominal numbers translate into a 5.3 year post-launch lifetime. In a worst-case scenario where all of the engineering uncertainties stack up unfavorably, the lifetime would drop to 4.0 years, which still compares quite well with the mission minimum lifetime requirement of 2.7 years. By regulating the power dissipation to accommodate the telescope temperature requirements of the instrument that is in use, we are expecting at least an additional four months of cryogenic lifetime beyond the nominal 5.3 years ${ }^{13}$. Additional information on the measured on-orbit thermal performance of the Spitzer CTA can be found in Finley et al. ${ }^{14}$ Following the depletion of the cryogens, the telescope will warm up, but it will still be colder than the outer shell. Current estimates suggest that 
the telescope temperature will always be $<30 \mathrm{~K}$. At this temperature, both the instrumental background and the detector dark current should be low enough for Spitzer to continue natural background-limited operations in the shortest wavelength IRAC bands at 3.6 and $4.5 \mu \mathrm{m}$.

\subsection{Pointing performance}

In general the Spitzer observatory is realizing pointing performance that is better than the pre-launch predictions. The star tracker in particular has proven to be very accurate, with a noise-equivalent-angle of approximately 0.11 arc-seconds using an average of 35 tracked stars. Further details on the in-flight performance of the Spitzer star trackers can be found elsewhere in these proceedings ${ }^{15}$. The cryo-mechanical variation in alignment over time between the star tracker mounted on the warm spacecraft and the cold telescope bore-sight has proven to be very small and easily calibrated, so that the star tracker can be used to directly point the telescope bore-sight to better than 0.5 arc-second, 1- $\sigma$ RMS radial uncertainty. The thermal stability has also allowed for more infrequent measurements (twice per day as opposed to the pre-launch plan of three times per day) of the star tracker and telescope's bore-sight alignment, which directly translates into increased science operations efficiency. The PCRS meets its star centroiding accuracy requirement of 0.14 arcseconds, 1- $\sigma$ RMS radial uncertainty ${ }^{16}$. For offset movements less than $30 \sim$ arcminutes, the PCS meets the 0.4 arcsecond offset accuracy requirement. It takes less than 150 seconds for the telescope to slew 15 degrees and to settle to its commanded position to within the above tolerances. Once the telescope pointing has settled, it is stable to within

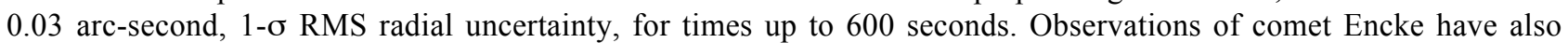
demonstrated that the PCS is able to track moving solar objects at rates up to at least 0.14 arc-seconds per second.

The on-orbit angular momentum that is built up on from a combination of solar pressure and uncompensated helium venting has proven to have a rate that is less than was predicted. During the design of the observatory care was taken to align the vector of solar pressure with the center of mass as closely as possible, and efforts were made to balance the thrust from the two CTA helium vent nozzles so that each would counteract the effects of the other. These efforts were well-rewarded, in that they have resulted in very low momentum build-up rate and a low frequency - once or twice per week - of actuations of the reaction control system to remove angular momentum. In spite of some pre-launch concerns

about leaking RCS thruster valves, the on-orbit performance of these components has been excellent ${ }^{3}$. As a result, the $\mathrm{N}_{2}$ gas in the RCS system should last for more than 10 years.

\subsection{Telemetry performance}

The Spitzer onboard telemetry hardware has performed flawlessly with the exception of one timing problem in the design of one of the spacecraft communication circuit boards. This problem was known before launch and manifests itself very rarely, but does result in the loss of a very small amount of science data when it occurs. To date there has been only one missed DSN pass, which was due to a malfunction in the DSN ground station and not the Spitzer flight hardware or software. The data from this missed pass was successfully retrieved in subsequent downlink sessions.

\subsection{Operations performance}

To date the Spitzer spacecraft has been reasonably free from operational anomalies, allowing it to realize its high science return. Details of the in-orbit checkout and science validation period operations can be found in Miles et al. ${ }^{17}$ As of May 19, 2004, the spacecraft has entered safe mode five times, with a summary of the causes given in Table 3.

Spitzer encountered a very large solar proton storm beginning 2003 October 28. During the two-day period of this storm the observatory received an integrated dose of $1.6 \times 10^{9} \mathrm{p}^{+}-\mathrm{cm}^{-2}$ for proton energies greater than $50 \mathrm{MeV}$, roughly the integrated dose expected under normal conditions over the first 2.5 years of operations. During the course of the storm the science instruments were powered off. Aside from a small number $(<4 \%)$ of IRS detector pixels that exhibited increased dark current, a correctible change in the offset bias of the wide-angle sun sensors on the spacecraft, and a very slight decrease in the efficiency of its solar arrays, the observatory was unaffected by this event. 
Table 3

Spitzer Safing Events

Spitzer Safing Event Entry Date

August 25, 2003

August 27, 2003

November 12, 2003

December 30, 2003

December 31, 2003
Cause and Resolution

High background in the star tracker fields due to high levels of earthshine shortly after launch caused a failure to correctly acquire the spacecraft attitude. This problem resolved itself once Spitzer moved further away from the earth.

High bearing friction in one of the reaction wheel assemblies caused by stiff lubricants caused excess current draw. The spacecraft bus heater temperature set-points were increased to eliminate this problem.

An error in the algorithm that checks for changes in the system momentum caused a fault to be declared during an observatory slew. Updating the algorithm with the correct coefficients fixed this problem.

A wheel zero-speed crossing in one of the reactions wheels during a long slow observatory mapping observation slew led to stiction in the bearings, which was reported as a fault. Steps were taken to ensure that significant momentum was distributed among all four reaction wheel assemblies before any such future observations of this type were made.

An error in the safemode recovery procedure that handles updating the onboard ephemeris caused the pointing control system to declare a fault. The procedure has been changed to avoid such problems in the future.

\subsection{Science instrument performance}

The of Spitzer focal-plane science instruments are all working and returning excellent-quality science data. A plot of the realized on-orbit sensitivities is shown in Figure 3. An up-to-date listing of the Spitzer instruments' performance can always be found at the Spitzer Science Center web site. ${ }^{18}$ A top-level summary of the instruments' status is given below.

\subsubsection{IRAC:}

All four bands of the IRAC instrument are working well and producing valid science data. The two shortest wavelength bands, Bands 1 and 2, have signal-to-noise sensitivities that are approximately 20\% better than was predicted based on the ground-based test data. Band 3 is approximately $23 \%$ less sensitive than was predicted and the Band 4 sensitivity is essentially the same as had been predicted. All of these data are for 200 second integration times on point-sources. The $\mathrm{S} / \mathrm{N}$ for all four IRAC bands improves as the square-root of the integration time once the background limit is achieved when this occurs depends on the band and the region of sky being observed. At very long integration times confusion caused by sources in the sky background sets a limit to the sensitivity - again this limit depends on the region of sky being observed. The variations from the predicted sensitivities are probably due to the difficulty in reproducing the extremely low background of the Spitzer environment in the ground test facilities. It should be emphasized that even in Band 3 the IRAC sensitivity is orders of magnitude higher than any instrument that has been available to date.

The measured on-orbit image quality of the IRAC is excellent, with total wavefront errors less than $\lambda / 20$ in each of the bands. Diffuse stray light from scattered zodiacal light does appear in the arrays at a low level, and the effects are more pronounced in Bands 1 and 2 than they are in Bands 3 and 4. There are also small regions just outside of the IRAC 
fields-of-view that give rise to specular glints - the observation planning tools at the SSC will help the prudent observer to avoid placing bright objects in these regions.

The IRAC detector arrays have proven to be very stable over time; observations of calibrations standards have varied by less than $2 \%$ over the mission. The arrays are affected by cosmic ray hits at the rate of one per second per array, about the predicted rate. The cosmic rays only influence the behavior of a few pixels around the hit location and the effects completely disappear in the subsequent frames. Latent images are visible in the arrays after a bright source has occupied the same region of the array for a long period of time. This effect is most pronounced in Bands 1 and 4 and can be mitigated by dithering the sky image around on the array. Thermal annealing the arrays completely removes any latent images and therefore as a result the frequency of these anneals has been increased above the rate that had been planned before launch.

In summary, the IRAC instrument is performing at least as well as had been predicted. Further details of the on-orbit performance of the IRAC can be found in Hora, et al. ${ }^{19}$ in this volume and in the paper by Fazio et al. in the dedicated issue of the Astrophysical Journal Supplement Series devoted to the Spitzer mission's early results. ${ }^{1}$

\subsubsection{IRS:}

All four modules of the IRS instrument are working well and producing valid science data. In addition to performing spectroscopy, the IRS peak-up arrays have demonstrated that they can be used for imaging, which can be particularly useful in the case of the blue peak-up array since the bandpass of this channel is located between the longest wavelength channel of the IRAC and the shortest wavelength channel of the MIPS. The on-orbit responsivity of all four detector arrays has been found to exceed the pre-launch estimates that were based on ground test data and analysis, the two long wavelength modules' arrays by a factor of two and the two short wavelength modules' arrays by a factor of 1.5. Most of this increase is due to design margin that was not used up. The measured array noises were the same as in the ground tests so that the increase in responsivity will translate directly into an increase in sensitivity. It should be emphasized however that array pixelization effects limit the signal-to-noise that can be achieved in many cases. In general, Spitzer was designed to achieve extremely high sensitivities on very faint sources. As a results the IRS instrument arrays undersample the optical point-spread function in order to minimize the effects of detector read noise. This under-sampling can make it more difficult to apply proper flat-field corrections, which translates into additional noise in a spectrum. For observations of faint sources this additional noise is not significant, but for those observations of brighter objects where very high signal-to-noise is desired this noise source can easily dominate, and does not integrate down with additional integration time. At present is appears that the maximum signal-to-noise that can be obtained is somewhere between 50:1 and 100:1.

The IRS arrays have proven to be extremely stable over time, with the responsivity varying less than $1 \%$ over the months that the IRS has been operating on-orbit. As was mentioned in Section 2.5 above, the IRS instrument was affected by the large solar storm of October, 2003. This damage manifested itself as increased dark current in a small number of pixels in the IRS arrays, and this damage appears to be permanent. The number of pixels is small enough however that it has had very little effect on the IRS performance. It should be noted that since the IRS arrays see a much smaller background than the detectors on the Spitzer imaging instruments do, they are much more affected by an increase in dark current. During ground testing it was noticed that one of the order blocking filters in the IRS Long-Low module appeared to have suffered delamination during a thermal cycle. The extent of this damage has stayed constant during the high launch-induced vibration environment. It does seriously affect the IRS Long-Low data, but on-orbit measurements found no evidence for out-of-band blue leaks, indicating that they are less than $5 \%$ for a Rayleigh-Jeans source.

In summary the IRS instrument is performing better on-orbit than had been predicted before launch. Further details of the on-orbit performance of the IRS can be found in Houck, et al. ${ }^{20}$ in this volume and in the paper by Houck et al. in the dedicated issue of the Astrophysical Journal Supplement Series devoted to the Spitzer mission's early results. ${ }^{1}$

\subsubsection{MIPS:}

All three of the MIPS detector arrays are alive and returning valid science data, although some sections of the $70 \mu \mathrm{m}$ and $160 \mu \mathrm{m}$ arrays suffer from some cabling problems, as depicted in Figures 4 and 5. The $24 \mu \mathrm{m}$ channel has been 
measured to have a sensitivity that is approximately a factor of 1.5 better than was predicted based on ground-based tests and analysis. As with the IRS this was due to under-utilized design margin. This array has also exhibited excellent stability since launch, with photometry of standards repeatable to within 1\%. As shown in Figure 4 the MIPS $70 \mu \mathrm{m}$ array has a good half and a bad half. The sensitivity of the good half is approximately a factor of three worse than was predicted pre-launch, largely due to higher-than-expected high-energy cosmic ray hit rates and long time-constant effects in its Ge:Ga detectors. Further details of these effects can be found in the paper by Rieke et al. in the dedicated issue of the Astrophysical Journal Supplement Series devoted to the Spitzer mission's early results. ${ }^{1}$ The $160 \mu \mathrm{m}$ channel has also exhibited a sensitivity that is down by a factor of approximately three from the pre-launch predictions, again due to cosmic rays and long time constant effects. It should be emphasized that these reductions in sensitivity are applicable to only the shorter integration times in very clear areas of the sky. With the relatively large diffractionlimited beam sizes in the MIPS instrument, plus the fact that extragalactic background sources generally exhibit increasing flux levels with wavelength in this infrared regime, the MIPS becomes confusion noise-limited in many scientific applications. In the case of the $70 \mu \mathrm{m}$ channel it reaches its confusion limit in about half an hour, while the $160 \mu \mathrm{m}$ channel reaches the confusion limit in just a few minutes. Thus at 70 and 160um MIPS quickly reaches the fundamental sensitivity achievable by an $85-\mathrm{cm}$ telescope. It should be additionally emphasized that even with some non-functioning array elements that the MIPS instrument provides Spitzer with far-infrared imaging capabilities far in excess of those available in any previous facilities.

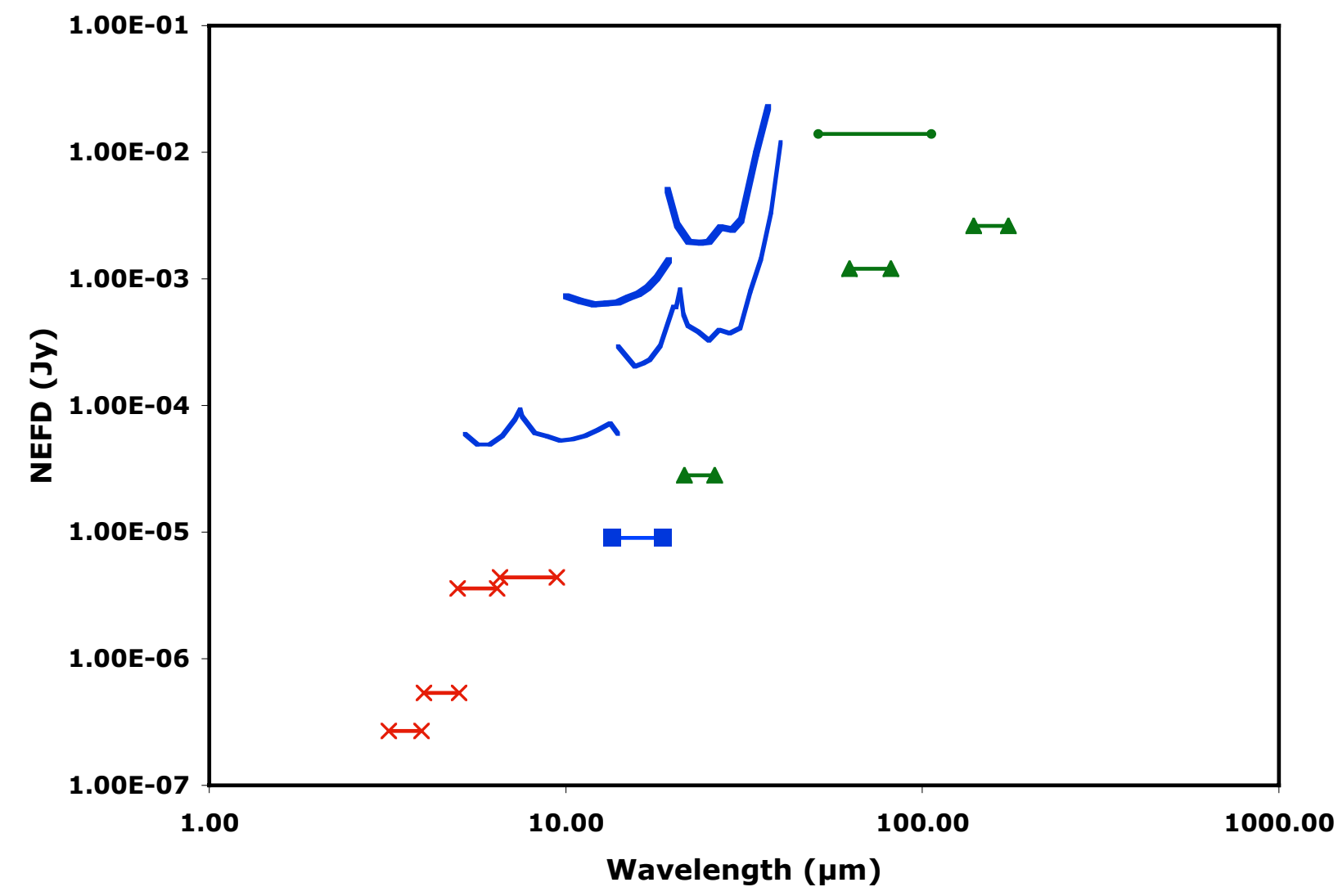

Figure 3. The measured on-orbit point-source sensitivity and wavelength coverage of the Spitzer science instruments. All of the sensitivities are for 500 second integration times and are 1- $\sigma$. In most cases the realized sensitivity can depend strongly on the zodiacal emission level and background confusion. The sensitivities quoted here are for the most optimal locations in the sky and in the case of the MIPS are for the better half of the $70 \mu \mathrm{m}$ array. The key for the lines in the figure is: red lines with the cross endpoints - IRAC instrument sensitivity; blue line with the filled-square endpoints - the IRS peakup array when it is used for imaging; green lines with the filled-triangle endpoints - MIPS instrument sensitivity; green line with the filled-circle endpoints - MIPS SED mode sensitivity; thin blue lines - IRS low-resolution sensitivity; thick blue lines - IRS high-resolution sensitivity. 


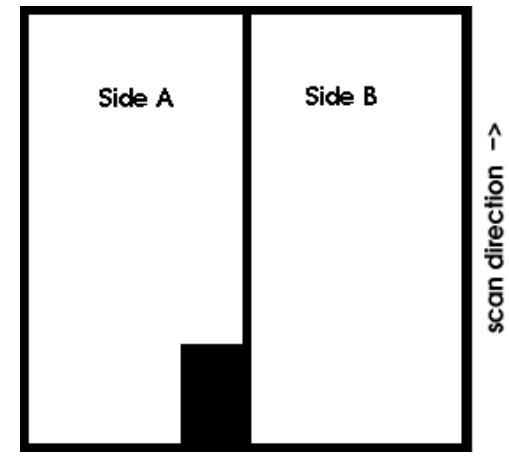

Figure 4. A diagram of the $32 \times 32$ MIPS $70 \mu \mathrm{m}$ array. The array is divided into two $16 \times 32$ pixel halves. A partial short in the cabling to Side B that developed during the on-orbit CTA cool down has resulted in significantly increased noise in this side, such that for most scientific purposes this half of the array cannot be used. Cabling problems have also led to an inoperative $4 \mathrm{x} 8$ section of Side A, shown in the figure.

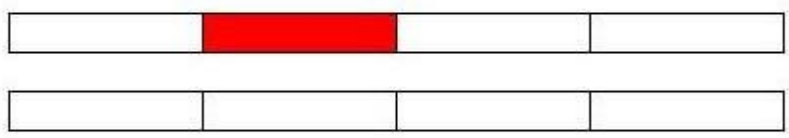

Figure 5. A diagram of the $2 \times 20$ MIPS $160 \mu \mathrm{m}$ array. One $1 \times 5$ pixel section indicted above is inoperative due to a cabling problem to one of the readouts.

The image quality of MIPS instrument is excellent, as can be seen in the $24 \mu \mathrm{m}$ image in Figure 3 . The $160 \mu \mathrm{m}$ channel has a short-wavelength filter leak that admits some $1.6 \mu \mathrm{m}$ light that must be accounted for when observing blue objects. Further details of the on-orbit performance of the MIPS can be found in Rieke, et al. ${ }^{21}$ in this volume and in the paper by Rieke et al. in the dedicated issue of the Astrophysical Journal Supplement Series devoted to the Spitzer mission's early results. ${ }^{1}$

\section{CONCLUSIONS}

Following a 90-day in-orbit checkout and scientific validation period, the Spitzer Space Telescope entered normal operations mode and has been returning excellent scientific data. With few exceptions, the on-orbit observatory systems have been performing as predicted or better, which has allowed Spitzer to realize its potential for scientific return.

\section{ACKNOWLEDGMENTS}

The Spitzer Space Telescope is operated by the Jet Propulsion Laboratory, California Institute of Technology, USA under NASA contract 1407. T. Roellig would like to acknowledge the support of the NASA Office of Space Sciences.

\section{REFERENCES}

1. Various papers describing the first science results from the Spitzer observatory can be found in a dedicated issue of The Astrophysical Journal Supplement Series, 154, 2004.

2. $\quad$ D. B. Gallagher, "The development and mission of the Spitzer Space Telescope", Astronomical Telescopes and Instrumentation - Proc. SPIE, 5487-01 (this issue), 2004.

3. K. C. Patel \& S. R. Spath, "Spitzer Space Telescope: observatory description and performance", Astronomical Telescopes and Instrumentation - Proc. SPIE, 5487-81 (this issue), 2004 
4. D. B. Gallagher, W. R. Irace, \& M. W. Werner, "Development of the Space Infrared Telescope Facility (SIRTF)", SPIE Proceedings, 4850, pp 17-29, 2003.

5. D. J. Tenerelli, A. Klavins, J. Miles, T. Decker, W. W. Sable, \& J. Fanson, "The Spitzer Space Telescope thermally stable spacecraft structure", Astronomical Telescopes and Instrumentation - Proc. SPIE, 5487-96 (this issue) 2004.

6. A. K. Mainzer, E. T. Young, T. P. Greene, N. Acu, T. H. Jamieson, H. Mora, S. Sarfati, \& R. W. van Bezooijen, "Pointing Calibration and Reference Sensor for the Space Infrared Telescope Facility", SPIE Proceedings, 3356, pp 1095-1101, 1998.

7. G. G. Fazio, J. L. Hora, S. P. Willner, J. R. Stauffer, M. N. L. Ashby, Z. Wang, E. V. Tollestrup, J. Pipher, W. Forrest, C. McCreight, C. H. Moseley, W. F. Hoffman, P. Eisenhardt, \& E. L. Wright, "The Infrared Array Camera (IRAC) for the Space Infrared Telescope Facility (SIRTF)", SPIE Proceedings, 3354, pp 1024-1031, 1998.

8. J. R. Houck, T. L. Roellig, J. Van Cleve, B. Brandl, \& K. Uchida, "IRS: the spectrograph on the SIRTF; its fabrication and testing", SPIE Proceedings, 4131, pp 70-377, 2000.

9. E. T. Young, G. H. Rieke, J. Vadien, H. Dole, C. Englebracht, K. D. Gordon, G. B. Heim, D. M. Kelly, \& J. A. Stansberry, "Ground test characterization of the Multiband Imaging Photometer for SIRTF (MIPS)", SPIE Proceedings, 4850, pp 98-107, 2003.

10. J. H. Kwok, M. Garcia, E. Bonfiglio, \& S. Long, "Spitzer Space Telescope mission design”, Astronomical Telescopes and Instrumentation - Proc. SPIE, 5487-89 (this issue), 2004.

11. W. F. Hoffmann, J. L. Hora, J. E. Mentzell, C. T. Marx, P. R. Eisenhardt, S. J. Carey, \& S. T. Megeath, "Determination of Spitzer Space Telescope focus from IRAC images without a focus slew", Astronomical Telescopes and Instrumentation - Proc. SPIE, 5487-88 (this issue), 2004.

12. R. D. Gehrz, E. A. Romana, W. F. Hoffmann, J. P. Schwenker. J. E. Mentzell, J. L. Hora, P. R. Eisenhardt, B. R. Brandl, L. Armus, K. R. Stapelfeldt, D. C. Hines, A. K. Mainzer, E,. T. Young, \& D. G. Elliott, "The state of the focus and image quality of the Spitzer Space Telescope as measured in orbit", Astronomical Telescopes and Instrumentation - Proc. SPIE, 5487-86 (this issue), 2004.

13. C. R. Lawrence, "Optimizing cryogen utilization on the Spitzer Space Telescope", Astronomical Telescopes and Instrumentation - Proc. SPIE, 5487-82 (this issue), 2004.

14. P. T. Finley, R. A. Hopkins, and R. B. Schweickart, "Flight cooling of the Spitzer Space Telescope Cryogenic Telescope Assembly", Astronomical Telescopes and Instrumentation - Proc. SPIE, 5487-02 (this issue) 2004.

15. R. W. H. van Bezooijen, "Flight performance of the Spitzer Space Telescope autonomous star trackers", Astronomical Telescopes and Instrumentation - Proc. SPIE, 5487-94 (this issue), 2004.

16. A. K. Mainzer, E. T. Young, \& D. S. Swanson, "On-orbit performance testing of the Pointing Calibration and Reference Sensor for the Spitzer Space Telescope”, Astronomical Telescopes and Instrumentation - Proc. SPIE, 548707 (this issue), 2004.

17. J. W. Miles, S. H. Linick, S. Long, J. Gilbert, M. Garcia, C. Boyles, M. Werner, \& R. K. Wilson, "Execution of the Spitzer in-orbit checkout and science verification plan", Astronomical Telescopes and Instrumentation - Proc. SPIE, $\mathbf{5 4 8 7 - 8 3}$ (this issue), 2004.

18. http://ssc.spitzer.caltech.edu.

19. J. L. Hora, et al., "In-flight performance and calibration of the infrared array camera (IRAC) for the Spitzer Space Telescope", Astronomical Telescopes and Instrumentation - Proc. SPIE, 5487-06 (this issue) 2004.

20. J. R. Houck, et al., "The in-flight performance of the infrared spectrograph", Astronomical Telescopes and Instrumentation - Proc. SPIE, 5487-05 (this issue), 2004.

21. G. H. Rieke, et al., "On-orbit performance of the MIPS instrument", Astronomical Telescopes and Instrumentation - Proc. SPIE, 5487-04 (this issue), 2004. 